\title{
Al Does not Have to Be Too Complicated or Expensive for Your Business
}

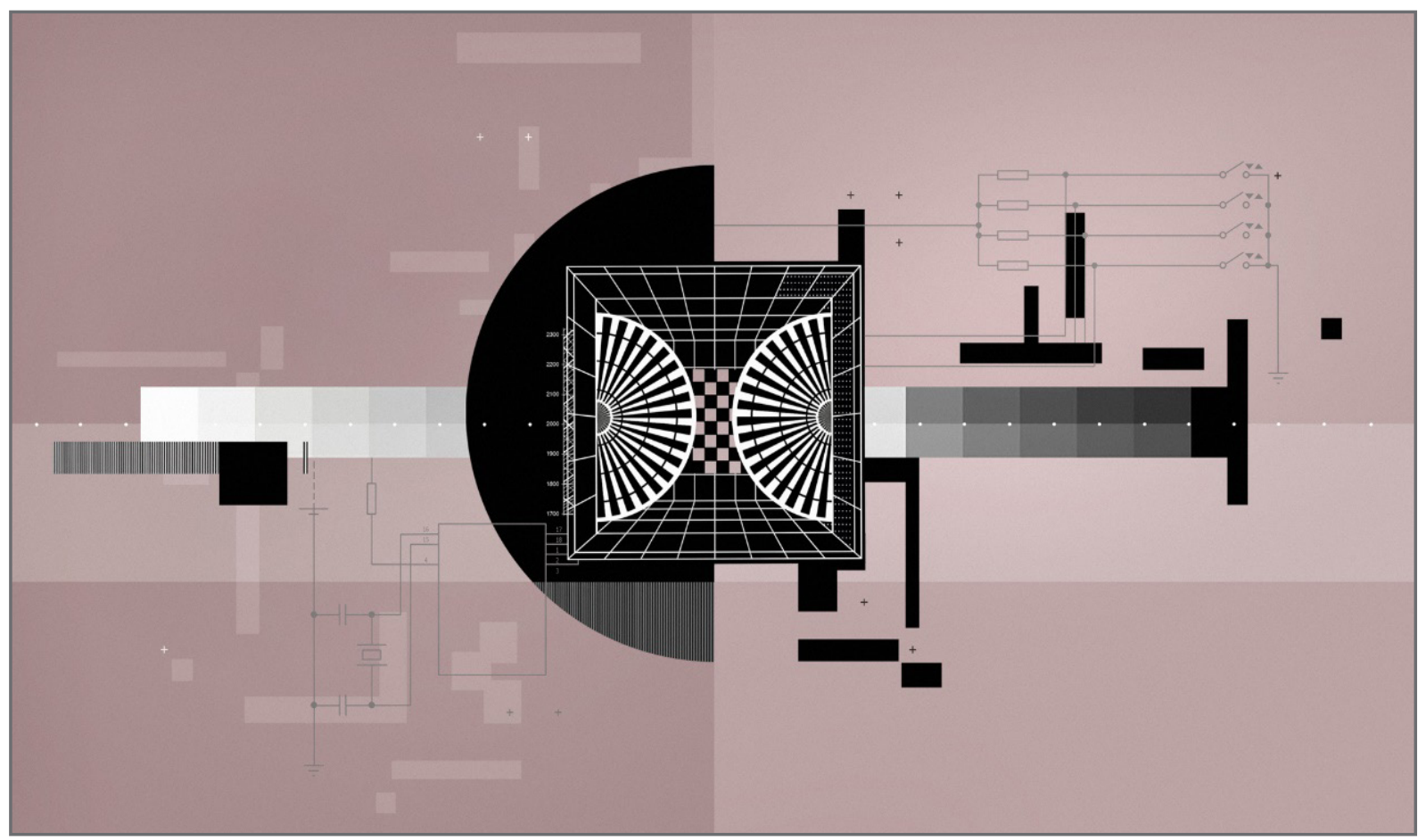

Summary. For most companies that are interested in using $\mathrm{Al}$, there isn't a clear model to follow. The approach to building Al used by massive internet companies like Amazon and Google just doesn't translate - most companies don't have overflowing troves of data they can...more Despite the vast potential of artificial intelligence (Al), it hasn't caught hold in most industries. Sure, it has transformed consumer internet companies such as Google, Baidu, and Amazon - all massive and data-rich with hundreds of millions of users. But for projections that $\mathrm{Al}$ will create $\$ 13$ trillion of value a year to come true, industries such as manufacturing, agriculture, and healthcare still need to find ways to make this technology work for them. Here's the problem: The playbook that these consumer internet companies use to build their Al systems - where a single one-size-fits-all Al system can serve massive numbers of users - won't work for these other industries. Instead, these legacy industries will need a large number of bespoke solutions that are adapted to their many diverse use cases. This doesn't mean that Al won't work for these industries, however. It just means they need to take a different approach. To bridge this gap and unleash Al's full potential, executives in all industries should adopt a new, data-centric approach to building Al. Specifically, they should aim to build Al systems with careful attention to ensuring that the data clearly conveys what they 
need the Al to learn. This requires focusing on data that covers important cases and is consistently labeled, so that the Al can learn from this data what it is supposed to do. In other words, the key to creating these valuable Al systems is that we need teams that can program withdata rather than program with code. Why adopting Al outside of tech can be so hard Why isn't Al widely used outside consumer internet companies? The top challenges facing $\mathrm{Al}$ adoption in other industries include: 1. Small datasets. In a consumer internet company with huge numbers of users, engineers have millions of data points that their Al can learn from. But in other industries, the dataset sizes are much smaller. For example, can you build an $\mathrm{Al}$ system that learns to detect a defective automotive component after seeing only 50 examples? Or to detect a rare disease after learning from just $100 \mathrm{di}-$ agnoses? Techniques built for 50 million data points don't work when you have only 50 data points. 2. Cost of customization. Consumer internet companies employ dozens or hundreds of skilled engineers to build and maintain monolithic Al systems that create tremendous value - say, an online ad system that generates more than $\$ 1$ billion in revenue per year. But in other industries, there are numerous \$1-5 million projects, each of which needs a custom Al system. For example, each factory manufacturing a different type of product might require a custom inspection system, and every hospital, with its own way of coding health records, might need its own Al to process its patient data. The aggregate value of these hundreds of thousands of these projects is massive; but the economics of an individual project might not support hiring a large, dedicated Al team to build and maintain it. This problem is exacerbated by the ongoing shortage of Al talent, which further drives up these costs. 3. Gap between proof of concept and production. Even when an Al system works in the lab, a massive amount of engineering is needed to deploy it in production. It is not unusual for teams to celebrate a successful proof of concept, only to realize that they still have another 12-24 months of work before the system can be deployed and maintained. For Al to realize its full potential, we need a systematic approach to solving these problems across all industries. The data-centric approach to Al, supported by tools designed for building, deploying, and maintaining $\mathrm{Al}$ applications - called machine learning operations (MLOps) platforms - will make this possible. Companies that adopt this approach faster will have a leg up relative to competitors. Data-centric Al development Al systems are made up of software - the computer program that includes an $\mathrm{Al}$ model - and data, the information used to train the model. For example, to build an $\mathrm{Al}$ system for automated inspection in manufacturing, an Al engineer might create software that implements a deep learning algorithm, that is then shown a dataset comprising pictures of good and defective parts, so it can learn to distinguish between them. Over the last decade, a lot of $\mathrm{Al}$ research was driven by software-centric development (also called modelcentric development) in which the data is fixed, and teams attempt to optimize or invent new programs to learn well from the available data. Many tech companies had large datasets from millions of consumers, and they used it to drive a lot of innovation in Al. But at Al's current level of sophistication, the bottleneck for many applications is getting the right data to feed to the software. We've heard about the benefits of big data, but we now know that for many applications, it is more fruitful to focus on making sure we have good data - data that clearly illustrates the concepts we need the Al to learn. This means, for example, the data should be reasonably comprehensive in its coverage of important cases and labeled consistently. Data is food for Al, and modern Al systems need not only calories, but also high-quality nutrition. 
Shifting your focus from software to data offers an important advantage: it relies on the people you already have on staff. In a time of great Al talent shortage, a data-centric approach allows many subject matter experts who have vast knowledge of their respective industries to contribute to the $\mathrm{Al}$ system development. For example, most factories have workers that are highly skilled at defining and identifying what counts as a defect (is a $0.2 \mathrm{~mm}$ scratch a defect? or is it so small that it doesn't matter?). If we expect each factory to ask its workers to invent new Al software as a way to get that factory the bespoke solution it needs, progress will be slow. But we instead build and provide tools to empower these domain experts to engineer the data - by allowing them to express their knowledge about manufacturing through providing data to the Al - their odds of success will be much higher. Make building and using Al systematic and repeatable The shift toward data-centric Al development is being enabled by the emerging field of MLOps, which provides tools that make building, deploying, and maintaining $\mathrm{Al}$ systems easier than ever before. Tools that are geared to help produce highquality datasets, in particular, hold the key to addressing the challenges of small datasets, high cost of customization, and the long road to getting an Al project into production outlined above. How, exactly? First, ensuring high-quality data means that Al systems will be able to learn from the smaller datasets available in most industries. Second, by making it possible for a business' domain experts, rather than Al experts, to engineer the data, the ability to use Al will become more accessible to all industries. And third, MLOps platforms provide much of the scaffolding software needed to take an Al system to production, so teams no longer have to develop this software. This allows teams to deploy Al systems - and bridge the gap between proof of concept and production weeks or months rather than years. The vast majority of valuable Al projects have yet to be imagined. And even for projects that teams are already working on, the gap that leads to deployment in production remains to be bridged - indeed, Accenture estimates that $80 \%$ to $85 \%$ of companies' $\mathrm{Al}$ projects are in the proof-of-concept stage. Here're some things companies can do right now: 1. Instead of merely focusing on the quantity of data you collect, also consider the quality, make sure it clearly illustrates the concepts we need the Al to learn. 2. Make sure your team considers taking a data-centric approach rather than a software-centric approach. Many Al engineers, including many with strong academic or research backgrounds, were trained to take a software-centric approach; urge them to adopt data-centric techniques as well. 3. For any $\mathrm{Al}$ project that you intend to take to production, be sure to plan the deployment process and provide MLOps tools to support it. For example, even while building a proof of concept system, urge the teams to begin developing a longer-term plan for data management, deployment, and $\mathrm{Al}$ system monitoring and maintenance. It's possible for Al to become a thriving asset outside of data-rich consumer internet businesses, but has yet to hit its stride in other industries. But because of this, the greatest untapped opportunity for Al may lie in taking it to these other industries. Just as electricity has transformed every industry, $\mathrm{Al}$ is on a path to do so too. But the next few steps on that path will require a shift in our playbook for how we build and deploy Al systems. Specifically, a new data-centric mindset, coupled with MLOps tools that allow industry domain experts to participate in the creation, deployment and maintenance of $\mathrm{Al}$ systems, will ensure that all industries can reap the rewards that Al can offer. 